\title{
A AMOSTRAGEM NA AVALIAÇÃO DAS LIXAS-DO-COQUEIRO'
}

\author{
MARIA DE LOURDES DA SILVA LEAL ${ }^{2}$ e DULCE REGINA NUNES WARWICK ${ }^{3}$
}

\begin{abstract}
RESUMO - A avaliação das doenças foliares do coqueiro (Cocos nucifera L.), conhecidas como lixagrande e lixa-pequena (verrugoses), causadas por Sphaeredothis acrocomiae e Phyllachora torrendiella, respectivamente, depara-se com o problema do método de amostragem, uma vez que não existe um método consensual em uso. Este trabalho foi realizado com o objetivo de comparar dois métodos de coleta de amostras mais utilizados na avaliação dessas doenças: método A: coleta de seis folíolos/planta, em uma única folha; método $\mathrm{B}$ : coleta de seis folíolos/planta, um em cada folha. $\mathrm{O}$ estudo foi desenvolvido a partir de três amostras de 300 folíolos (dez plantas x cinco folhas x seis folíolos), coletados em três genótipos de coqueiro, nos quais foi determinado o número de estromas da lixa-grande e da lixapequena. Com base nesses dados, estimaram-se as variâncias de folhas dentro de plantas e folíolos dentro de folhas e plantas, necessárias para os cálculos das estimativas das médias amostrais nos dois métodos em comparação, além de outras alternativas formuladas. Em ambas as lixas, em todos os genótipos, as estimativas da variância da média amostral calculadas pelo método A foram superiores às calculadas pelo método B, o que comprova que este último é mais eficaz que o primeiro. Outros tamanhos de amostra também foram avaliados e comparados ao método B, e constatou-se que amostras de seis folhas/planta, coletando-se, em cada uma, dois ou três folíolos, reduzem a variância amostral em $20 \%$ ou $30 \%$, respectivamente, podendo, portanto, ser utilizadas com mais eficiência.
\end{abstract}

Termos para indexação: Cocos nucifera, doenças das plantas, fungos, folhas, métodos de análise.

\section{SAMPLING IN THE EVALUATION OF THE COCONUT VERRUCOSES}

\begin{abstract}
The evaluation of leaf diseases of coconuts, Cocos nucifera L., known as large verrucose and small verrucose, caused by Sphaerodothis acrocomiae and Phyllachora torrendiella, respectively, come upon the problem of sampling method. Since there is not a consensual method for measuring coconut verrucose incidence, the present work was done with the objective of comparing two sampling methods of common use: method A: sampling six leaflets/plant in a single leaf; method B: sampling six leaflets/plant, from different leaves. The study was developed starting from three samples of 300 leaflets (ten plants $\mathrm{x}$ five leaves $\mathrm{x}$ six leaflets) collected in three coconut genotypes, in which the stromata number of large verrucose and of small verrucose were counted. Throughout these data the variance was calculated from the leaf in the plant and the leaflet in the leaf and plant, necessary for the estimated sample average in the two compared methods, as well in the other formulated alternatives. For the two diseases, in all the genotypes, the estimate of variance of the sample mean calculated by the A method was higher than the one calculated by the B method, showing that the last method is more appropriate than the first one. Other sample sizes were also studied and compared by the B method, resulting that sample of two or three leaflets collected in six leaves per plant decreased sample variance on respectively $20 \%$ or $30 \%$, probably being of more accurate applicability.
\end{abstract}

Index terms: Cocos nucifera, plant diseases, fungi, leaves, analytical methods.

\footnotetext{
${ }^{1}$ Aceito para publicação em 13 de março de 2000.

${ }^{2}$ Eng. Agrôn., M.Sc., Embrapa-Centro de Pesquisa Agropecuária dos Tabuleiros Costeiros (CPATC), Caixa Postal 44, CEP 49001-970 Aracaju, SE. E-mail: lurdinha@cpatc.embrapa.br

${ }^{3}$ Eng. Agrôn., Ph.D., Embrapa-CPATC. E-mail: dulce@cpatc.embrapa.br
}

\section{INTRODUÇÃO}

As doenças foliares do coqueiro, conhecidas por lixa-grande e lixa-pequena, verrugoses causadas pelos fungos Sphaerodothis acrocomiae (Montagne) von Arx \& Müller (Joly, 1961) e Phyllachora torrendiella (Batista) (Subileau, 1993), provocam con- 
sideráveis perdas na produção dos coqueirais brasileiros, especialmente no Nordeste do Brasil. Vários trabalhos foram realizados para estudar diferentes aspectos das lixas, tais como o controle químico da lixa-pequena (Resende et al., 1988), o efeito da adubação sobre a incidência das lixas (Leal et al., 1994), e a avaliação de genótipos resistentes a elas (Leal et al., 1996), entre outros. Entretanto, nenhum estudo metodológico sobre a avaliação dessas doenças foi realizado, principalmente sobre métodos de amostragem.

Resende et al. (1988) coletaram amostras de seis folíolos da parte terminal de uma folha previamente marcada em cada planta, e nelas contaram o número de estromas da lixa-pequena. Leal et al. (1994) também utilizaram este método de amostragem para a avaliação do número de estromas das lixas. Entretanto, Leal et al. (1996) fizeram uso de dois métodos de amostragem: o acima descrito, de coleta de seis folíolos em uma folha determinada, e a amostragem de seis folíolos - um de cada uma das seis últimas folhas -, constatando que o primeiro método se mostrou ineficiente, e por isso não foi utilizado na obtenção dos resultados aos quais se propunha.

Este trabalho teve por objetivo comparar a eficiência dos dois métodos de amostragem na avaliação de cada uma das doenças.

\section{MATERIAL E MÉTODOS}

Foram coletados folíolos de três genótipos de coqueiro em duas áreas experimentais da EmbrapaCentro de Pesquisa Agropecuária dos Tabuleiros Costeiros (CPATC), nos municípios de Ilha das Flores e Umbaúba, no Estado de Sergipe, onde se constata alta incidência das lixas. No município de Ilha das Flores, a coleta foi realizada em agosto de 1997, na variedade anão-vermelho-da-malásia (AVM); em Umbaúba, as coletas foram feitas em setembro de 1997, na variedade anão-verde-de-jiqui (AVeJ) e no híbrido de Jiqui (AVeJ x gigante-do-brasil). Em cada variedade das duas áreas foram avaliadas dez plantas, e em cada planta, as cinco últimas folhas funcionais. De cada uma destas folhas, foram retirados, do terço médio, e alternadamente, ou seja, de um lado e outro da folha, seis folíolos, nos quais foi contado o número de estromas da lixa-grande e da lixa-pequena.
Inicialmente, procedeu-se a uma análise exploratória dos dados obtidos em cada variável, com a finalidade de se determinar o tipo de transformação mais adequada a eles. Posteriormente, os dados transformados foram submetidos à análise de variância, utilizando-se o modelo estatístico de classificação hierárquica, proposto por Snedecor (1970) e reportado por Lima \& Moraes (1985) e Rossetti et al. (1988), exposto a seguir:

$Y_{i j k}=\mu+\alpha_{i}+\beta_{i j}+\delta_{i j k}$ sendo $\mathrm{Y}_{\mathrm{ijk}} \mathrm{o}$ número de estromas contados no folíolo $\mathrm{k}(\mathrm{k}=1,2, \ldots, \mathrm{c})$, da folha $\mathrm{j}(\mathrm{j}=1,2, \ldots, \mathrm{b})$, na planta $\mathrm{i}$ $(\mathrm{i}=1,2, \ldots, \mathrm{a}) ; \mu$ a média geral; $\alpha_{\mathrm{i}}$ o efeito da planta $\mathrm{i}$; $\beta_{\mathrm{ij}}$ o efeito da folha $\mathrm{j}$ na planta $\mathrm{i}$, e $\delta_{\mathrm{ijk}} \mathrm{O}$ efeito do folíolo $\mathrm{k}$ na folha $\mathrm{j}$ e planta $\mathrm{i}$. Pressupõe-se, ainda, que $\alpha_{\mathrm{i}}, \beta_{\mathrm{ij}}$ e $\delta_{\mathrm{ijk}}$ são normalmente distribuídos com média zero e variâncias $\sigma_{\mathrm{a}}^{2}, \sigma_{\mathrm{b}}^{2} \mathrm{e} \sigma^{2}$, respectivamente.

Para determinar qual dos dois métodos de amostragem em uso, citados por Leal et al. (1996), era o mais eficiente, denominou-se método A aquele em que foi tomada uma única folha de cada planta $\mathrm{e}$ nela foram avaliados seis folíolos, independentemente do número de plantas amostradas. Do mesmo modo, designou-se como método B aquele em que foram tomadas seis folhas por planta, e em cada uma dessas folhas foi avaliado um folíolo. Para efeito dessa comparação, além de outras alternativas formuladas, foram analisados os dados coletados de seis folíolos de cada uma das últimas cinco folhas funcionais, de dez plantas, conforme acima mencionado. No modelo utilizado, considerou-se o efeito de planta fixo e os efeitos de folha e folíolo aleatórios. Assim, o esquema da análise de variância de cada amostra, de acordo com o modelo proposto, com $\alpha_{i}$ fixo, foi o apresentado na Tabela 1 , do qual deduz-se que a variância de $\delta_{\mathrm{ijk}}, \sigma^{2}$, é estimada por $\mathrm{s}^{2}=\mathrm{Q}_{2}$, a variância de $\beta_{\mathrm{ij}}$, $\sigma_{\mathrm{b}}^{2}$, é estimada por

$\mathrm{s}_{\mathrm{b}}^{2}=\left(\mathrm{Q}_{1}-\mathrm{Q}_{2}\right) / \mathrm{c}$ e a variância do número médio de estromas por planta, $\sigma^{2}\left(y_{i}\right)$, é estimada por $\mathrm{s}^{2}(\mathrm{y})=\mathrm{s}_{\mathrm{b}}{ }^{2} / \mathrm{b}+\mathrm{s}^{2} / \mathrm{bc}$.

No presente caso, em que todas as amostras foram compostas de cinco folhas por planta e seis folíolos por folha, tem-se:

$\mathrm{s}^{2}=\mathrm{Q}_{2} \mathrm{e} \mathrm{s}_{\mathrm{b}}^{2}=\left(\mathrm{Q}_{1}-\mathrm{Q}_{2}\right) / 6$.

Considerando-se essas estimativas como referência, a estimativa da variância do número médio de 
estromas de uma amostra de seis folíolos, retirados de uma única folha (método A), seria:

$\mathrm{s}^{2}\left(\mathrm{y}_{\mathrm{A}}\right)=\mathrm{s}_{\mathrm{b}}^{2}+\mathrm{s}^{2} / 6=\mathrm{Q}_{1} / 6$,

ao passo que a estimativa da variância da média de uma amostra de seis folíolos, sendo um de cada folha (método B), seria:

$\mathrm{s}^{2}\left(\mathrm{y}_{\mathrm{B}}\right)=\mathrm{s}_{\mathrm{b}}^{2} / 6+\mathrm{s}^{2} / 6=\left(\mathrm{Q}_{1}+5 \mathrm{Q}_{2}\right) / 36$.

Neste modelo, porém, pressupõe-se $\mathrm{Q}_{1}>\mathrm{Q}_{2}$, para que se possa estimar $\mathrm{s}_{\mathrm{b}}{ }^{2}$, o que nem sempre ocorre. A estimativa da média amostral também não leva em conta a correlação que se pressupõe existir entre folíolos dentro da mesma folha. Portanto, pode-se considerar ainda o modelo mais geral, proposto por Pimentel-Gomes et al. (1963) e reportado por Rossetti et al. (1988) e Leal (1993):

$\mathrm{Y}_{\mathrm{ijk}}=\mu+\alpha_{\mathrm{i}}+\mathrm{e}_{\mathrm{ijk}}$,

tal que

$\mathrm{E}\left(\mathrm{e}^{2}{ }_{\mathrm{ijk}}\right)=\sigma^{2}$,

$\mathrm{E}\left(\mathrm{e}_{\mathrm{ijk}}, \mathrm{e}_{\left.\mathrm{i}^{\prime} \mathrm{j}^{\prime} \mathrm{k}^{\prime}\right)}\right)=0 \mathrm{e}$

$\mathrm{E}\left(\mathrm{e}_{\mathrm{ijk}}, \mathrm{e}_{\mathrm{ijk}} \mathrm{i}^{\prime}\right)=\rho \sigma^{2}$,

onde $\rho$ é o coeficiente de correlação intraclasse. As esperanças dos quadrados médios de folhas dentro de plantas e folíolos dentro de folhas, segundo Cochran \& Cox (1957), são mostradas na Tabela 2 , de onde se deduz que as estimativas de $\sigma^{2}$ e $\rho$ são, respectivamente:

$\mathrm{s}^{2}=\left[\mathrm{Q}_{1}+(\mathrm{c}-1) \mathrm{Q}_{2}\right] / \mathrm{c} \mathrm{e}$

$\mathrm{r}=\left(\mathrm{Q}_{1}-\mathrm{Q}_{2}\right) /\left[\mathrm{Q}_{1}+(\mathrm{c}-1) \mathrm{Q}_{2}\right]$.

Assim, com relação às amostras analisadas, temse:

$\mathrm{s}^{2}=\left(\mathrm{Q}_{1}+5 \mathrm{Q}_{2}\right) / 6 \mathrm{e}$

$\mathrm{r}=\left(\mathrm{Q}_{1}-\mathrm{Q}_{2}\right) /\left(\mathrm{Q}_{1}+5 \mathrm{Q}_{2}\right)$.

Neste caso, a estimativa da variância da média de uma amostra de seis folíolos, retirados de uma única folha, seria:

$s^{2}\left(y_{A}\right)=\left[s^{2}(1+5 \rho)\right] / 6=Q_{1} / 6$,

e a estimativa da variância da média de uma amostra de seis folíolos, retirados um de cada folha, seria:

$\mathrm{s}^{2}\left(\mathrm{y}_{\mathrm{B}}\right)=\mathrm{s}^{2} / 6=\left(\mathrm{Q}_{1}+5 \mathrm{Q}_{2}\right) / 36$.

Observa-se que as estimativas obtidas através dos dois modelos se equivalem para o caso de $\rho>0$, e que o segundo modelo é mais geral, admitindo $\mathrm{Q}_{1}<\mathrm{Q}_{2}$, no caso de $\rho<0$. Assim, em qualquer caso, foram adotadas as fórmulas acima para estimar as variâncias das médias amostrais, nos dois métodos de amostragem.

O método de amostragem mais eficiente será aquele que proporcionar uma menor variância do número médio de estromas por planta (Pimentel-Gomes et al.,

TABELA 1. Componentes de variância (quadrado médio e esperança matemática correspondente) do modelo misto de classificação hierárquica, com $\alpha_{i}$ fixo.

\begin{tabular}{llll}
\hline Causas da variação & G.L. & Q.M. & E(Q.M.) \\
\hline Plantas & $\mathrm{a}-1$ & & \\
Folhas dentro de plantas & $\mathrm{a}(\mathrm{b}-1)$ & $\mathrm{Q}_{1}$ & $\sigma^{2}+c \sigma_{\mathrm{b}}{ }^{2}$ \\
Folíolos dentro de folhas e plantas & $\mathrm{ab}(\mathrm{c}-1)$ & $\mathrm{Q}_{2}$ & $\sigma^{2}$ \\
\hline
\end{tabular}

TABELA 2. Componentes de variância (quadrado médio e esperança matemática correspondente) do modelo misto de classificação hierárquica, considerando o coeficiente de correlação intraclasse $\rho$.

\begin{tabular}{llll}
\hline Causas da variação & G.L. & Q.M. & E(Q.M.) \\
\hline Plantas & $\mathrm{a}-1$ & & \\
Folhas dentro de plantas & $\mathrm{a}(\mathrm{b}-1)$ & $\mathrm{Q}_{1}$ & $\sigma^{2}[1+(\mathrm{c}-1) \rho]$ \\
Folíolos dentro de folhas e plantas & $\mathrm{ab}(\mathrm{c}-1)$ & $\mathrm{Q}_{2}$ & $\sigma^{2}(1-\rho)$ \\
\hline
\end{tabular}


1963; Rossetti et al., 1986), o que fornecerá, invariavelmente, menor coeficiente de variação.

\section{RESULTADOS E DISCUSSÃO}

Nas Tabelas 3 e 4 são apresentados os quadrados médios das análises de variância, bem como as estimativas das médias, dos componentes de variância e dos coeficientes de correlação intraclasse referentes aos dados de número de estromas da lixagrande e lixa-pequena, transformados em $\log (x+10)$, obtidos nos três genótipos, que serviram de base para os cálculos do presente trabalho.

Com base na análise dos dados transformados do número de estromas da lixa-grande na variedade AVM (Tabela 3), a estimativa da variância da média
$\left(\mathrm{s}^{2}\left(\mathrm{y}_{\mathrm{A}}\right)\right)$ de uma amostra obtida pelo método A seria de 0,5497 , ao passo que em uma amostra obtida pelo método $\mathrm{B}$ a estimativa $\mathrm{s}^{2}\left(\mathrm{y}_{\mathrm{B}}\right)$ seria de 0,1346 .

Como a média geral estimada para esse genótipo foi de 3,93, verifica-se que, pelo primeiro método, o coeficiente de variação seria de $18,86 \%$, enquanto com o segundo ele cairia para $9,34 \%$.

Ainda com base na análise dos dados da variedade AVM, no caso da lixa-pequena (Tabela 4), a amostragem de seis folíolos coletados de uma única folha forneceria uma variância da média amostral de 0,2903, o que daria, com uma média estimada em 3,29, um coeficiente de variação de $16,38 \%$.

Em uma amostra de seis folíolos, retirados um de cada folha, ter-se-ia s${ }^{2}\left(\mathrm{y}_{\mathrm{B}}\right)=0,0790$ e um coeficiente de variação de $8,54 \%$.

TABELA 3. Quadrado médio, média, componentes de variância $\left(\mathrm{s}^{2}\right.$ e $\left.\mathrm{s}_{\mathrm{b}}^{2}\right)$ e coeficiente de correlação intraclasse $(\mathbf{r})$, referentes aos dados de número de estromas da lixa-grande, transformados em $\log (x+10)$, obtidos nos genótipos anão-vermelho-da-malásia (AVM), anão-verde-de-jiqui (AVeJ) e híbrido de Jiqui.

\begin{tabular}{lrllc}
\hline Causas da variação & G.L. & \multicolumn{3}{c}{ Quadrado médio } \\
\cline { 3 - 5 } & & AVM & AVeJ & Híbrido de Jiqui \\
\hline Plantas & 9 & 3,7287 & 2,3792 & 5,2507 \\
Folhas dentro de plantas & 40 & 3,2982 & 0,6125 & 1,0034 \\
Folíolos dentro de folhas e plantas & 250 & 0,3098 & 0,1229 & 0,1869 \\
\hline Média geral & & 3,93 & 4,94 & 4,05 \\
$\mathrm{~s}^{2}$ & & 0,3098 & 0,1229 & 0,1869 \\
$\mathrm{~s}_{\mathrm{b}}{ }^{2}$ & 0,4981 & 0,0816 & 0,1361 \\
$\mathrm{r}$ & & 0,62 & 0,40 & 0,42 \\
\hline
\end{tabular}

TABELA 4. Quadrado médio, média, componentes de variância $\left(\mathrm{s}^{2}\right.$ e $\left.\mathrm{s}_{\mathrm{b}}{ }^{2}\right)$ e coeficiente de correlação intraclasse $(\mathbf{r})$, referentes aos dados de número de estromas da lixa-pequena, transformados em $\log (x+10)$, obtidos nos genótipos anão-vermelho-da-malásia (AVM), anão-verde-de-jiqui (AveJ) e híbrido de Jiqui.

\begin{tabular}{lrlcc}
\hline Causas da variação & G.L. & \multicolumn{3}{c}{ Quadrados médios } \\
\cline { 3 - 5 } & & AVM & AVeJ & Híbrido de Jiqui \\
\hline Plantas & 9 & 3,1800 & 10,0971 & 3,3898 \\
Folhas dentro de plantas & 40 & 1,7419 & 1,5426 & 0,9713 \\
Folíolos dentro de folhas e plantas & 250 & 0,2208 & 0,1860 & 0,1962 \\
\hline Média geral & & 3,29 & 4,72 & 3,82 \\
$\mathrm{~s}^{2}$ & 0,2208 & 0,1860 & 0,1962 \\
$\mathrm{~s}_{\mathrm{b}}^{2}$ & 0,2535 & 0,2261 & 0,1292 \\
$\mathrm{r}$ & & 0,53 & 0,55 & 0,40 \\
\hline
\end{tabular}


Tais resultados, bem como os de cálculos análogos relativos à variedade AveJ e ao híbrido de Jiqui, são apresentados na Tabela 5, onde se observa que o método B de amostragem proporcionaria um decréscimo significativo da variância amostral, e, conseqüentemente, do coeficiente de variação em relação ao método A. Resultados semelhantes foram obtidos por Lima \& Moraes (1985), que, ao estudar a avaliação do índice de infecção da ferrugem-do-cafeeiro, tendo a folha como unidade amostral, concluiu ser mais eficiente aumentar o número de plantas na amostra do que aumentar o número de folhas por planta. Pimentel-Gomes et al. (1963), em seu trabalho sobre a amostragem de cana-de-açúcar para determinações tecnológicas, também encontrou vantagens em se amostrar uma só cana por touceira em um maior número de touceiras, para um mesmo número de canas, exceto para a variável peso, em apenas uma variedade, em que os resultados foram semelhantes, independentemente do método amostral.

O coeficiente de variação médio estimado, em relação ao número de estromas da lixa-grande, considerando-se os três genótipos, obtido no método A, foi de $11,81 \%$. Admitindo-se esse valor, num experimento com cinco tratamentos e dez repetições (plantas), e se de cada planta fosse coletada uma amostra de seis folíolos retirados de determinada folha, terse-ia uma diferença mínima significativa a $5 \%$ de probabilidade pelo teste de Tukey, de 15,16\% da média. Pelo método B, o coeficiente de variação médio foi de $6,27 \%$, e a diferença mínima significativa, em condições semelhantes às referidas acima, cairia para $8,05 \%$ da média.
No caso da lixa-pequena, o coeficiente de variação médio dos três genótipos, no método $\mathrm{A}$, foi de $12,55 \%$, o que daria, em um experimento com cinco tratamentos e dez repetições, uma diferença mínima significativa a $5 \%$ de probabilidade, pelo teste de Tukey, de 16,11\% da média. Pelo método B de amostragem, em que o coeficiente de variação médio foi de $6,73 \%$, a diferença mínima significativa, em condições análogas, cairia para $6,73 \%$ da média.

Tais resultados mostram que o método B leva a uma maior precisão experimental, em relação ao método A, visto que propicia menores coeficientes de variação, e, conseqüentemente, maior precisão nas comparações de médias, e pode detectar menores diferenças entre médias de tratamentos.

Ainda com o intuito de comparar os diferentes tipos de métodos amostrais, foram tomadas as médias e estimativas médias dos componentes de variância médios dos três genótipos, $\mathrm{y}=4,31, \mathrm{~s}^{2}=0,2065 \mathrm{e}$ $\mathrm{s}_{\mathrm{b}}{ }^{2}=0,2386$, em relação à lixa-grande, e $\mathrm{y}=3,94$, $\mathrm{s}^{2}=0,2010$ e $\mathrm{s}_{\mathrm{b}}^{2}=0,2029$, em relação à lixa-pequena, e calcularam-se as estimativas da variância da média amostral e dos coeficientes de variação, variando-se o número de folhas e de folíolos até um limite de seis folhas x seis folíolos. Essas estimativas são apresentadas nas Tabelas 6 e 7, respectivamente, e sugerem que o aumento do número de folhas na amostra é sempre mais eficiente que o aumento do número de folíolos.

As razões entre as variâncias dos diferentes tamanhos de amostra e a variância da amostra mais eficiente em uso (método B) são mostradas nas Tabelas 8 e 9, em relação à lixa-grande e lixa-pequena,

TABELA 5. Estimativa da variância das médias amostrais e coeficientes de variação do número de estromas da lixa-grande e lixa-pequena, nos métodos A e B de amostragem.

\begin{tabular}{llccccc}
\hline \multirow{2}{*}{ Doença } & \multicolumn{1}{c}{ Genótipo } & \multicolumn{2}{c}{ Variância da média amostral } & & \multicolumn{2}{c}{ Coeficiente de variação (\%) } \\
\cline { 3 - 3 } \cline { 6 - 7 } & & Método A & Método B & & Método A & Método B \\
\hline \multirow{2}{*}{ Lixa-grande } & Anão-vermelho-da-malásia & 0,5497 & 0,1346 & & 18,86 & 9,34 \\
& Anão-verde-de-jiqui & 0,1021 & 0,0341 & & 6,47 & 3,74 \\
& Híbrido de Jiqui & 0,1672 & 0,0538 & & 10,10 & 5,73 \\
\hline \multirow{2}{*}{ Lixa-pequena } & Anão-vermelho-da-malásia & 0,2903 & 0,0790 & & 16,38 & 8,54 \\
& Anão-verde-de-jiqui & 0,2571 & 0,0687 & & 10,74 & 5,55 \\
& Híbrido de Jiqui & 0,1619 & 0,0542 & & 10,53 & 6,09 \\
\hline
\end{tabular}


TABELA 6. Estimativa da variância $\left(\mathrm{s}^{2}\right)$ das médias amostrais, e coeficiente de variação $(\mathrm{CV}$, \%) do número de estromas da lixa-grande, em diferentes tamanhos de amostra.

\begin{tabular}{|c|c|c|c|c|c|c|c|c|c|c|c|c|}
\hline \multirow{3}{*}{$\begin{array}{c}\text { № } \\
\text { de } \\
\text { folhas }\end{array}$} & \multicolumn{12}{|c|}{ Número de folíolos } \\
\hline & \multicolumn{2}{|c|}{1} & \multicolumn{2}{|c|}{2} & \multicolumn{2}{|c|}{3} & \multicolumn{2}{|c|}{4} & \multicolumn{2}{|c|}{5} & \multicolumn{2}{|c|}{6} \\
\hline & $\mathrm{s}^{2}$ & $\mathrm{CV}$ & $\mathrm{s}^{2}$ & $\mathrm{CV}$ & $s^{2}$ & $\mathrm{CV}$ & $s^{2}$ & $\mathrm{CV}$ & $s^{2}$ & $\mathrm{CV}$ & $\mathrm{s}^{2}$ & $\mathrm{CV}$ \\
\hline 1 & 0,4451 & 15,48 & 0,3418 & 13,56 & 0,3074 & 12,86 & 0,2902 & 12,50 & 0,2799 & 2,27 & 0,2730 & 12,12 \\
\hline 2 & 0,2225 & 10,94 & 0,1709 & 9,59 & 0,1537 & 9,10 & 0,1451 & 8,84 & 0,1399 & 8,68 & 0,1365 & 8,57 \\
\hline 3 & 0,1484 & 8,94 & 0,1139 & 7,83 & 0,1025 & 7,43 & 0,0967 & 7,22 & 0,0933 & 7,09 & 0,0910 & 7,00 \\
\hline 4 & 0,1113 & 7,74 & 0,0854 & 6,78 & 0,0768 & 6,43 & 0,0725 & 6,25 & 0,0699 & 6,14 & 0,0682 & 6,06 \\
\hline 5 & 0,0890 & 6,92 & 0,0684 & 6,07 & 0,0615 & 5,75 & 0,0580 & 5,58 & 0,0560 & 5,49 & 0,0546 & 5,42 \\
\hline 6 & 0,0742 & 6,32 & 0,0597 & 5,54 & 0,0512 & 5,25 & 0,0484 & 5,10 & 0,0467 & 5,01 & 0,0455 & 4,95 \\
\hline
\end{tabular}

TABELA 7. Estimativa da variância $\left(\mathrm{s}^{2}\right)$ das médias amostrais, e coeficiente de variação $(\mathrm{CV}$, \%) do número de estromas da lixa-pequena, em diferentes tamanhos de amostra.

\begin{tabular}{|c|c|c|c|c|c|c|c|c|c|c|c|c|}
\hline \multirow{3}{*}{$\begin{array}{c}\mathrm{N}^{\mathrm{o}} \\
\mathrm{de} \\
\text { folhas }\end{array}$} & \multicolumn{12}{|c|}{ Número de folíolos } \\
\hline & \multicolumn{2}{|c|}{1} & \multicolumn{2}{|c|}{2} & \multicolumn{2}{|c|}{3} & \multicolumn{2}{|c|}{4} & \multicolumn{2}{|c|}{5} & \multicolumn{2}{|c|}{6} \\
\hline & $\mathrm{s}^{2}$ & $\mathrm{CV}$ & $\mathrm{s}^{2}$ & $\mathrm{CV}$ & $\mathrm{s}^{2}$ & $\mathrm{CV}$ & $\mathrm{s}^{2}$ & $\mathrm{CV}$ & $\mathrm{s}^{2}$ & $\mathrm{CV}$ & $\mathrm{s}^{2}$ & $\mathrm{CV}$ \\
\hline 1 & 0,4039 & 16,13 & 0,3030 & 13,98 & 0,2699 & 13,19 & 0,2532 & 12,77 & 0,2431 & 12,51 & 0,2364 & 12,34 \\
\hline 2 & 0,2019 & 11,40 & 0,1510 & 9,88 & 0,1349 & 9,32 & 0,1266 & 9,03 & 0,1215 & 8,85 & 0,1182 & 8,72 \\
\hline 3 & 0,1346 & 9,31 & 0,1010 & 8,07 & 0,0900 & 7,61 & 0,0844 & 7,37 & 0,0810 & 7,22 & 0,0788 & 7,12 \\
\hline 4 & 0,1009 & 8,06 & 0,0757 & 6,98 & 0,0675 & 6,59 & 0,0633 & 6,38 & 0,0608 & 6,26 & 0,0591 & 6,17 \\
\hline 5 & 0,0808 & 7,21 & 0,0606 & 6,25 & 0,0540 & 5,90 & 0,0506 & 5,71 & 0,0486 & 5,60 & 0,0473 & 5,52 \\
\hline 6 & 0,0673 & 6,58 & 0,0505 & 5,70 & 0,0450 & 5,38 & 0,0422 & 5,21 & 0,0405 & 5,11 & 0,0394 & 5,04 \\
\hline
\end{tabular}

TABELA 8. Razão entre as variâncias de diferentes tamanhos de amostra e a variância da média amostral do número de estromas da lixa-grande em seis folhas e um folíolo por folha (método B).

\begin{tabular}{ccccccc}
\hline \multirow{2}{*}{$\begin{array}{c}\text { No de } \\
\text { folhas }\end{array}$} & \multicolumn{6}{c}{ Número de folíolos } \\
\cline { 2 - 7 } & 1 & 2 & 3 & 4 & 5 & 6 \\
\hline 1 & 6,00 & 4,61 & 4,14 & 3,91 & 3,77 & 3,68 \\
2 & 3,00 & 2,30 & 2,07 & 1,95 & 1,88 & 1,83 \\
3 & 2,00 & 1,53 & 1,38 & 1,30 & 1,26 & 1,22 \\
4 & 1,50 & 1,15 & 1,03 & 0,98 & 0,94 & 0,92 \\
5 & 1,20 & 0,92 & 0,83 & 0,78 & 0,75 & 0,73 \\
6 & 1,00 & 0,80 & 0,69 & 0,65 & 0,63 & 0,61 \\
\hline
\end{tabular}

respectivamente. No caso da lixa-grande (Tabela 8), verifica-se que a amostra de 20 folíolos (quatro folhas x cinco folíolos) forneceria uma variância $6 \%$ menor em relação ao método usual, ao passo que em uma amostra de cinco folhas e quatro folíolos/folha, a variância seria $22 \%$ menor. Resultados semelhan-
TABELA9. Razão entre as variâncias de diferentes tamanhos de amostra e a variância da média amostral do número de estromas da lixa-pequena em seis folhas e um folíolo por folha (método B).

\begin{tabular}{ccccccc}
\hline \multirow{2}{*}{$\begin{array}{c}\text { No de } \\
\text { folhas }\end{array}$} & \multicolumn{6}{c}{ Número de folíolos } \\
\cline { 2 - 7 } & 1 & 2 & 3 & 4 & 5 & 6 \\
\hline 1 & 6,00 & 4,50 & 4,01 & 3,76 & 3,61 & 3,51 \\
2 & 3,00 & 2,24 & 2,00 & 1,88 & 1,80 & 1,76 \\
3 & 2,00 & 1,50 & 1,34 & 1,25 & 1,20 & 1,17 \\
4 & 1,50 & 1,12 & 1,00 & 0,94 & 0,90 & 0,88 \\
5 & 1,20 & 0,90 & 0,80 & 0,75 & 0,72 & 0,70 \\
6 & 1,00 & 0,75 & 0,68 & 0,63 & 0,60 & 0,58 \\
\hline
\end{tabular}

tes foram constatados com relação à lixa-pequena (Tabela 9).

Constata-se, também, que, em ambas as lixas (Tabelas 8 e 9), resultados mais eficientes que o obtido pelo método $\mathrm{B}$ de amostragem seriam alcançados se fossem tomadas amostras de 16 ou mais folíolos em 
quatro folhas, dez ou mais folíolos em cinco folhas, ou ainda, 12 ou mais folíolos no caso de seis folhas. Entretanto, considerando-se o custo da amostragem de cada folíolo e a eficiência relativa obtida em cada caso, constata-se que amostras de 12 ou 18 folíolos em seis folhas, que forneceriam reduções de $20 \%$ e $30 \%$ na variância da média, respectivamente, seriam os mais recomendáveis.

\section{CONCLUSÕES}

1. Em ambas as lixas, o método de amostra de seis folíolos por planta, sendo um folíolo de cada folha (método B), é mais eficiente que a amostra de seis folíolos por planta, coletados de uma única folha (método A).

2. O acréscimo do número de folhas na amostra é mais eficiente que o acréscimo do número de folíolos por folha.

3. Maior eficiência na amostragem é obtida adotando-se amostras de seis folhas por planta, coletando-se dois ou três folíolos por folha.

\section{REFERÊNCIAS}

COCHRAN, W.G.; COX, G.M. Experimental designs. 2.ed. New York : J. Wiley, 1957. 585p.

JOLY, P. Le genre Sphaerodothis Sherr. Research Council of Israel Bulletin, Jerusalem, v.10D, p.187-193, 1961.

LEAL, E.C.; RAM, C.; WARWICK, D.R.N.; LEAL, M. de L. da S.; RENARD, J.L. Comportamento de híbridos de coqueiro Cocos nucifera em relação à lixapequena Phillachora torrendiella e à lixa-grande Sphaerodothis acrocomiae. Fitopatologia Brasileira, Brasília, v.21, n.3, p.325-327, 1996.

LEAL, E.C.; SANTOS, Z.G. dos; RAM, C.; WARWICK, D.R.N.; LEAL, M. de L. da S.; RENARD, J.L. Efeito da adubação mineral sobre a incidência das lixas Sphaerodothis torrendiella e Sphaerodothis acrocomiae no coqueiro Cocos nucifera $\mathrm{L}$.
Oléagineux, Montpellier, v.49, n.5, p.213-220, 1994.

LEAL, M. de L. da S. Otimização do tamanho de parcelas em experimentos com coqueiro gigante. Oléagineux, Montpellier, v.48, n.10, p.407-413, 1993.

LIMA, P.C.; MORAES, R.S. Método de amostragem para a avaliação do índice de infecção da ferrugem do cafeeiro (Hemileia vastatrix, BERK e BR). In: SIMPÓSIO DE ESTATÍSTICA APLICADA À EXPERIMENTAÇÃO AGRONÔMICA, 1.; REUNIÃO ANUAL DA REGIÃO BRASILEIRA DA SOCIEDADE INTERNACIONAL DE BIOMETRIA, 30., 1985, Piracicaba. Anais. Piracicaba : Fundação Cargill, 1985. p.124-139.

PIMENTEL-GOMES, F.; VALSECHI, O.; ABREU, C.P.; OLIVEIRA, E.R. A amostragem da cana-de-açúcar para determinações tecnológicas. Anais da Escola Superior de Agricultura Luís de Queiroz, Piracicaba, v.20, p.89-114, 1963.

RESENDE, M.L.V. de; COELHO, J.A.; BEZERRA, J.L. Seleção de fungicidas para o controle da lixa-pequena do coqueiro. Revista Theobroma, Itabuna, v.18, n.3, p.217-223, 1988.

ROSSETTI, A.G.; PEREIRA, A.V.; PIMENTEL-GOMES, F. A amostragem na experimentação em viveiro de seringueira. Pesquisa Agropecuária Brasileira, Brasília, v.21, n.8, p.837-841, ago. 1986.

ROSSETTI, A.G.; PIMENTEL-GOMES, F.; VIEGAS, R.M.F. Tamanho ótimo de parcela para experimentos em viveiro de seringueira. Pesquisa Agropecuária Brasileira, Brasília, v.23, n.6, p.621-625, jun. 1988.

SNEDECOR, G.W. Métodos estadísticos aplicados a la investigación agrícola y biológica. México : Continental, 1970. 626p.

SUBILEAU, C. Systématique et biologie du complexe parasitaire constitué du Phyllachora torrendiella (Bat.) nov. comb. et du Botryosphaeria cocogena nov. sp., agents fongiques du dessèchement foliaire du cocotier au Brésil. Paris : Université Paris 6, 1993. 121p. Thèse de Doctorat. 